\title{
Editorial
}

\section{Compactness Conditions in the Theory of Nonlinear Differential and Integral Equations}

\author{
Józef Banaś, ${ }^{1}$ Mohammad Mursaleen, ${ }^{2}$ Beata Rzepka, ${ }^{1}$ and Kishin Sadarangani ${ }^{3}$ \\ ${ }^{1}$ Department of Mathematics, Rzeszów University of Technology, Al. Powstanców, Warszawy 8, 35-959 Rzeszów, Poland \\ ${ }^{2}$ Department of Mathematics, Aligarh Muslim University, Aligarh 202 002, India \\ ${ }^{3}$ Departamento de Matematicas, Universidad de Las Palmas de Gran Canaria, Campus de Tafira Baja, \\ 35017 Las Palmas de Gran Canaria, Spain \\ Correspondence should be addressed to Józef Banaś; jbanas@prz.edu.pl
}

Received 10 January 2013; Accepted 10 January 2013

Copyright (C) 2013 Józef Banaś et al. This is an open access article distributed under the Creative Commons Attribution License, which permits unrestricted use, distribution, and reproduction in any medium, provided the original work is properly cited.

The concept of the compactness appears very frequently in explicit or implicit form in many branches of mathematics. Particulary, it plays a fundamental role in mathematical analysis and topology and creates the basis of several investigations conducted in nonlinear analysis and the theories of functional, differential, and integral equations. In view of the wide applicability of the mentioned branches, the concept of the compactness is one of the most useful in several topics of applied mathematics, engineering, mathematical physics, numerical analysis, and so on.

Reasoning based on concepts and tools associated with the concept of the compactness is very often used in fixed point theory and its applications to the theories of functional, differential, and integral equations of various types. Moreover, that concept is also applied in general operator theory. It is worthwhile mentioning several types of operators defined with help of the compactness, both in strong and in weak topology.

This special issue is devoted to discussing some aspects of the above presented topics. We focus here mainly on the presentation of papers being the outcome of research and study associated with existence results concerning nonlinear differential, integral or functional differential, and functional integral equations. We describe roughly the results contained in the papers covering this special issue.

One of those results, contained in the paper of Q. Li and Y. $\mathrm{Li}$, is concerned with the existence of positive periodic solutions of a second-order functional differential equation with multiple delay. The investigations concerning that equation are located in the space of real functions defined, continuous and periodic (with a fixed period) on the real line. The main tool used in the proofs is the fixed point index theory in cones.

The paper of C. Lizama and J. C. Pozo presents also existence results and is devoted to proving the existence of mild solutions of a semilinear integrodifferential equation in a Banach space. The arguments used in the study are based on theory of semigroups and the technique of measures of noncompactness.

The paper by Z. Liu et al., which we are going to describe briefly, discusses existence results for an integral equation of Volterra type. The authors of that paper used some special measure of noncompactness and the fixed point theorem of Darbo type to obtain a result on the existence of asymptotic stable solutions of the equation in question.

A review article concerning infinite systems of differential equations in the so-called BK spaces presents a brief survey of existence results concerning those systems. The authors, $\mathrm{M}$. Mursaleen and A. Alotaibi, present results obtained with help of the technique of measures of noncompactness.

In this special issue two research articles concerning the existence of solutions of nonlinear fractional differential equations with boundary value problems are included. The paper authored by Y. Zhao et al. discusses a result on the existence of positive solutions of a nonlinear fractional integrodifferential equation with multipoint boundary value problem. The investigations are conducted in an ordered 
Banach space. The main tools used in argumentations are related to a fixed point theorem for operators which are strictly condensing with respect to the Kuratowski measure of noncompactness. The research article of $\mathrm{H}$. Ergören and A. Kiliçman deals with existence of solutions of impulsive fractional differential equations with closed boundary conditions. The results of that paper are obtained with help of a Burton-Kirk fixed point theorem for the sum of a contraction and a completely continuous operator.

The technique of measures of noncompactness also creates the main tool utilized in the paper of S. Xie devoted to the existence of mild solutions of nonlinear mixed type integrodifferential functional evolution equations with nonlocal conditions. Apart of the existence, the controllability of the mentioned solutions is also shown.

The issue contains also three papers being only loosely related to compactness conditions. The paper by H. Eltayeb and A. Kiliçman discusses approximate solutions of a nonlinear system of partial differential equations with help of Sumudu decomposition technique and with the use of Adomian decomposition method. The second paper of the mentioned kind, written by M. Masjed-Jamei et al., discusses some new estimates for the error of the Simpson integration rule. That study is conducted in the classical Lebesgue spaces $L^{1}[a, b]$ and $L^{\infty}[a, b]$. Finally, the paper of A. Kiliçman and R. Abazari is dedicated to discussing exact solutions of the Schrödinger-Boussinesq system. The authors of that paper construct solutions of the system in question in the form of hyperbolic or trigonometric functions.

This issue includes also a review paper by J. Banaś and K. Sadarangani presenting an overview of several methods involving compactness conditions. Those methods are mainly related to some fixed point theorems (Schauder, Krasnosel'skii-Burton, and Schaefer) and to the technique associated with measures of noncompactness. Examples illustrating the applicability of those methods are also included.

\section{Acknowledgments}

The guest editors of this special issue would like to express their gratitude to the authors who have submitted papers for consideration. We believe that the results presented in this issue will be a source of inspiration for researchers working in nonlinear analysis and related areas of mathematics.

Józef Banaś

Mohammad Mursaleen Beata Rzepka

Kishin Sadarangani 


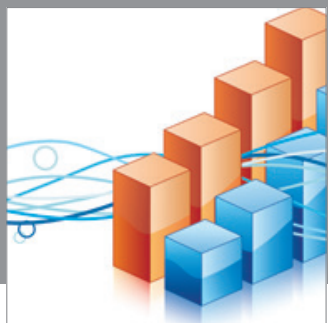

Advances in

Operations Research

mansans

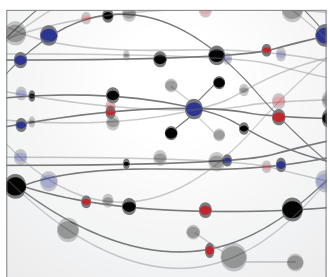

The Scientific World Journal
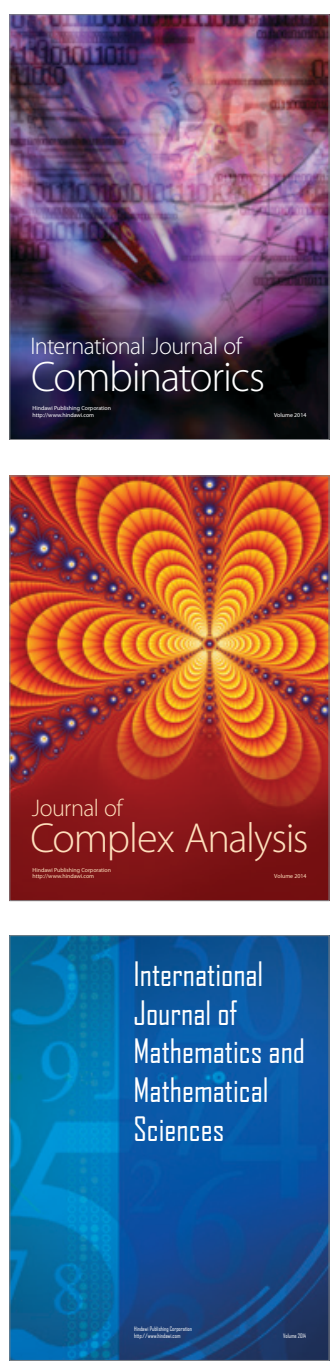
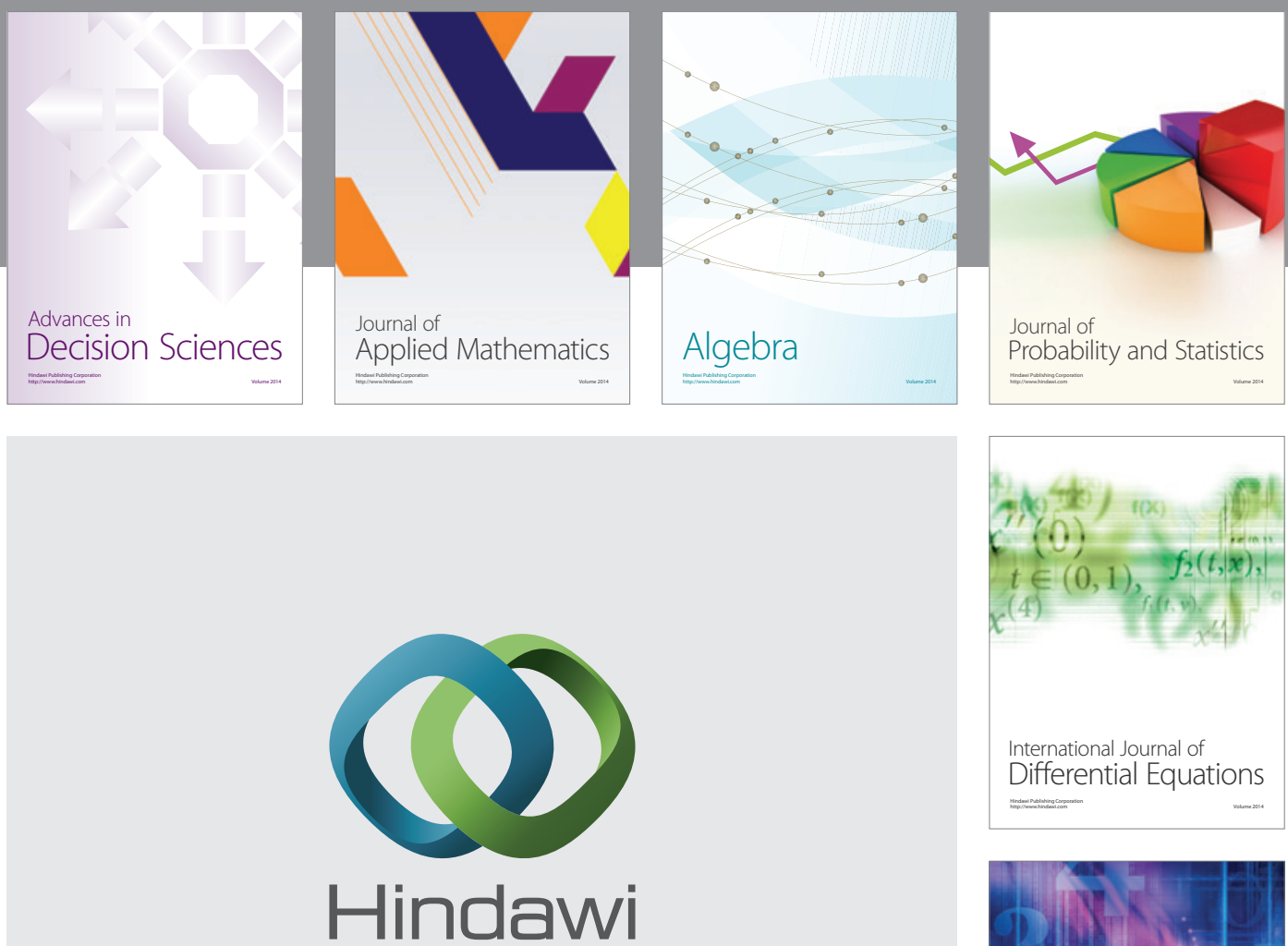

Submit your manuscripts at http://www.hindawi.com
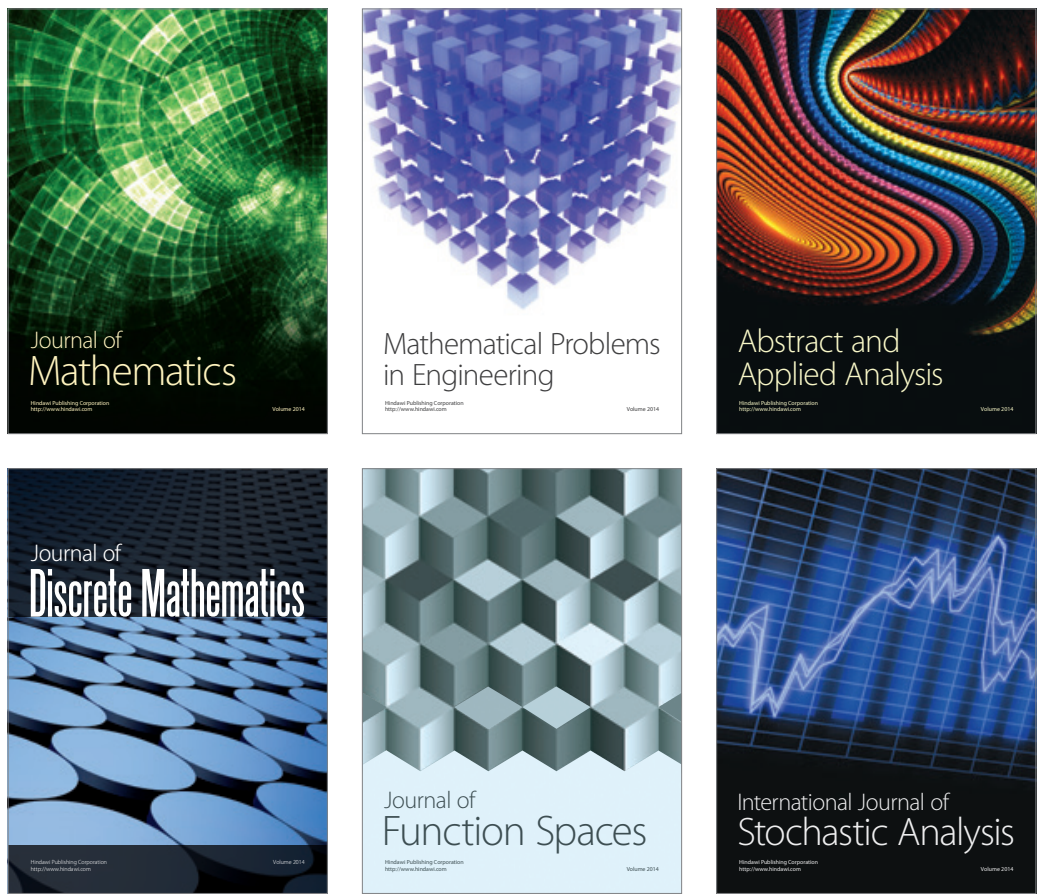

Journal of

Function Spaces

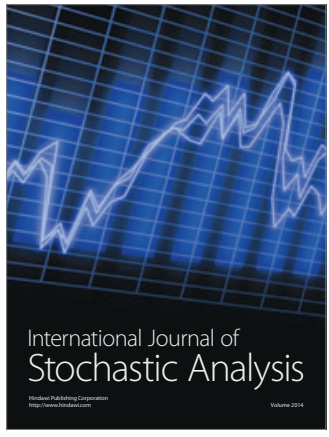

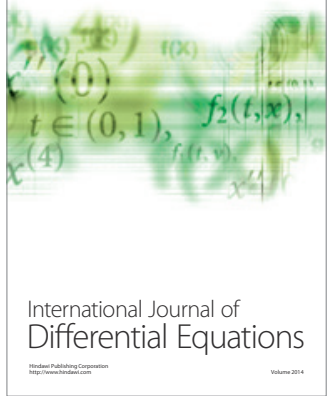
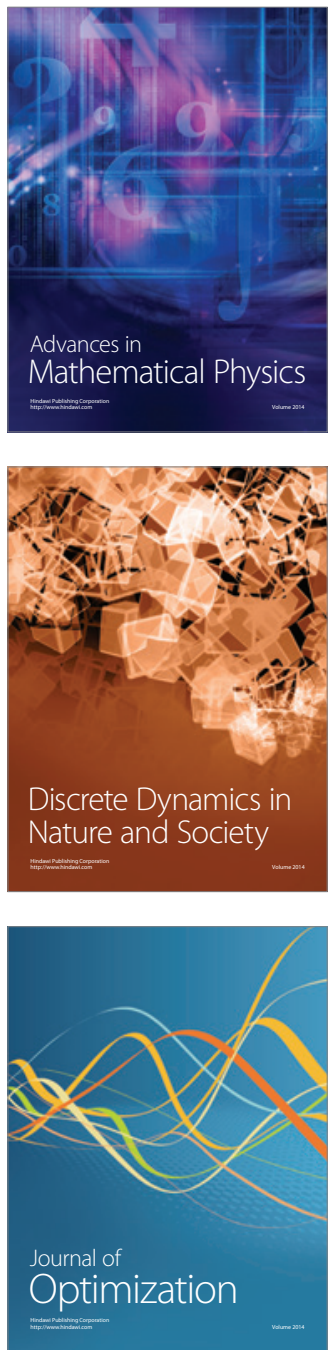